\title{
MALDI-TOF MS VERSUS 16S rRNA SEQUENCING: MINOR DISCREPANCY BETWEEN TOOLS IN IDENTIFICATION OF BACTEROIDES ISOLATES
}

\author{
KÁROly PÉTER SÁRVÁRI ${ }^{1}$, JÓZSEF SÓKI ${ }^{1}$, MiKLÓs IVÁN ${ }^{2}$, CECILIA MisZTI $^{3}$, \\ KrisZTINA LATKÓCZY ${ }^{4}$, SZILVIA ZSÓKA MELEGH ${ }^{5}$ and EDIT URBÁN ${ }^{1 *}$ \\ ${ }^{1}$ Institute of Clinical Microbiology, University of Szeged, Szeged, Hungary \\ ${ }^{2}$ Institute of Laboratory Medicine, Semmelweis University, Budapest, Hungary \\ ${ }^{3}$ Institute of Medical Microbiology, University of Debrecen, Debrecen, Hungary \\ ${ }^{4}$ SYNLAB Ltd., Budapest, Hungary \\ ${ }^{5}$ Institute of Medical Microbiology and Immunology, University of Pécs, Pécs, Hungary
}

(Received: 6 March 2017; accepted: 10 June 2017)

\begin{abstract}
Members of the genus Bacteroides are important components of the normal microbiota of gastrointestinal tract; however, as opportunistic pathogens are also associated with severe or even life-threatening infections with significant mortality. Various species within Bacteroides fragilis group are phenotypically very similar; thus, their identifications with traditional-automated biochemical methods are frequently inaccurate. The identification of the newly discovered or reclassified bacteria can be doubtful because of the lack of biochemical profile in the database of these tests. The aim of this study was to determine the accuracy of matrix-assisted laser desorption/ionization time-of-flight mass spectrometry (MALDI-TOF MS) method by testing of 400 Hungarian Bacteroides clinical isolates. Inaccurate identification results with MALDI-TOF MS were confirmed by $16 \mathrm{~S}$ rRNA gene sequencing and findings were compared with traditional-automated biochemical test rapid ID 32A method as well.
\end{abstract}

Keywords: Bacteroides fragilis group, sequencing, rapid ID 32A

\section{Introduction}

Although members of the Bacteroides genus are components of the normal microbiota of human gut, these strains are frequently isolated from sepsis, skin and soft tissue, intraabdominal and postoperative wound infections, pelvic, brain, and lung abscesses with the mortality rate of more than $19 \%$ [1-4]. In the infections caused by Bacteroides isolates, the most frequently used antibiotics are

\footnotetext{
*Corresponding author; E-mail: urban.edit@med.u-szeged.hu
} 
beta-lactam/beta-lactamase inhibitors, cephamycins, carbapenems, clindamycin, fourth-generation fluoroquinolones, and nitroimidazoles [1]. In the last 20 years, increasing antibiotic resistance rate of Bacteroides isolates was reported against cefoxitin, clindamycin, and moxifloxacin; however, amoxicillin/clavulanic acid, piperacillin/tazobactam, carbapenems, metronidazole, and tigecycline remained very active drugs against these bacteria [5]. Due to various antibiotic resistance patterns of species within the genus, adequate identification of Bacteroides isolates is very important to achieve optimal antimicrobial therapy [6]. Presumptive phenotypic identification based on colony morphology, antibiotic disks ( $5 \mu \mathrm{g}$ vancomycin, $1,000 \mu \mathrm{g}$ kanamycin, and $10 \mu \mathrm{g}$ colistin), spot tests, and microscopic morphology (Gram-stained smear) may provide information about the bacteria at genus or species level [4, 7]. For species-level identification, commercial biochemical tests are available detecting either preformed enzymes under aerobic environment or inducible enzymes under anaerobic conditions. The main disadvantages of phenotypic identification methods are that they cannot distinguish surely the closely related species, and the anaerobic bacteria grow relatively slowly, and some species are biochemically inactive $[3,6]$. In the last decades, remarkable changes in the taxonomy and nomenclature of anaerobic bacteria have been documented, owing to the development in molecular methods, new genera and species have been described. Identification of these new species by phenotypic methods can be difficult due to the lack of their biochemical profiles in various commercially available databases [8]. 16S rRNA gene sequencing is considered to be the gold standard method for identification; however, the application in routine diagnostic laboratory is difficult because of its time-consuming, expensive, and technically challenging features $[3,9]$. Among the molecular methods, the matrix-assisted laser desorption/ ionization time-of-flight mass spectrometry (MALDI-TOF MS) provides accurate and fast identification results of Bacteroides species [3]. The aim of this study was to test the accuracy of MALDI-TOF MS identification system using 400 Hungarian Bacteroides clinical isolates and to retest the strains with contradictory results given using MALDI-TOF in different laboratory in contradictory cases, rapid ID 32A (bioMérieux, France), and 16S rRNA gene sequencing methods were applied.

\section{Materials and Methods}

\section{Bacterial strains}

A total of 400 Bacteroides clinical isolates received from five Hungarian clinical microbiological centers (two centers from Budapest, Debrecen, Szeged, and Pécs) were collected between 2014 and 2016. The strains were stored at 
$-80{ }^{\circ} \mathrm{C}$ in cryobank vials with brain-heart infusion medium and with $20 \%$ glycerol until use. Local laboratories cultured and identified the examined strains according to standard laboratory operating procedures for anaerobic bacteria. The first identification was performed by MALDI-TOF MS (Bruker Daltonik, Germany) in the participating clinical microbiological centers. Species were distributed as follows during the first routine identification: 233 Bacteroides fragilis, 5 Bacteroides caccae, 34 Bacteroides ovatus, 69 Bacteroides thetaiotaomicron, 2 Bacteroides salyersiae, 14 Bacteroides uniformis, 27 Bacteroides vulgatus, 1 Bacteroides nordii, 14 Parabacteroides distasonis, and 1 Parabacteroides goldsteinii (Table I). Before the final MALDI-TOF analysis in Szeged, all the examined 400 strains were cultured on Schaedler agar (bioMérieux) for $48 \mathrm{~h}$ at $37{ }^{\circ} \mathrm{C}$ in anaerobic chamber (PerkinElmer, UK) under anaerobic conditions $\left(85 \% \mathrm{~N}_{2}, 10 \% \mathrm{CO}_{2}\right.$, and $\left.5 \% \mathrm{H}_{2}\right)$.

\section{MALDI-TOF MS}

The strains were identified in each center and also in Szeged by MALDI-TOF MS (Bruker Daltonik) with Biotyper version 3.0 software containing 5,989 mass spectra of reference strains of aerobic, anaerobic bacteria, and fungi. The reidentification of each strain in Szeged was performed by one person with three simultaneous measurements and three persons repeated it with one measurement per person with the same conditions (strains and chemicals). Results with the best log score values were accepted. The measurement mode was microflex, the parameters were:

Table I. Distribution of different Bacteroides species identified by MALDI-TOF MS $(n=400)$ in the original laboratory and reidentification in the laboratory of Szeged

\begin{tabular}{|c|c|c|c|c|c|c|c|c|c|c|}
\hline Species & $\begin{array}{c}\text { ID } \\
\text { Budapest I }\end{array}$ & $\begin{array}{c}\text { RD } \\
\text { Budapest I }\end{array}$ & $\begin{array}{c}\text { ID } \\
\text { Budapest II }\end{array}$ & $\begin{array}{c}\text { RD } \\
\text { Budapest II }\end{array}$ & $\begin{array}{c}\text { ID } \\
\text { Debrecen }\end{array}$ & $\begin{array}{c}\mathrm{RD} \\
\text { Debrecen }\end{array}$ & $\begin{array}{c}\text { ID } \\
\text { Szeged }\end{array}$ & $\mathrm{RD}$ & $\begin{array}{l}\text { ID } \\
\text { Pécs }\end{array}$ & $\mathrm{RD}$ \\
\hline B. fragilis & 49 & 48 & 52 & 49 & 63 & 64 & 60 & 60 & 9 & 9 \\
\hline B. caccae & 1 & 1 & 2 & 1 & 1 & 1 & 1 & 1 & - & - \\
\hline B. ovatus & 8 & 8 & 6 & 5 & 10 & 8 & 9 & 8 & 1 & 1 \\
\hline B. thetaiotaomicron & 14 & 13 & 23 & 24 & 19 & 24 & 13 & 13 & - & - \\
\hline B. salyersiae & - & - & 1 & 2 & 1 & - & - & - & - & - \\
\hline B. uniformis & 8 & 8 & - & - & 3 & 1 & 3 & 3 & - & - \\
\hline B. vulgatus & 11 & 10 & 6 & 6 & 2 & 1 & 8 & 8 & - & - \\
\hline P. distasonis & 8 & 9 & - & - & 1 & 1 & 5 & 5 & - & - \\
\hline $\begin{array}{l}\text { Bacteroides } \\
\text { cellulosilyticus }\end{array}$ & - & - & - & 1 & - & - & - & - & - & - \\
\hline $\begin{array}{c}\text { Bacteroides } \\
\text { stercoris }\end{array}$ & - & - & - & 1 & - & - & - & - & - & - \\
\hline P. goldsteinii & 1 & - & - & - & - & - & - & - & - & - \\
\hline B. nordii & - & 1 & - & 1 & - & - & 1 & 2 & - & - \\
\hline
\end{tabular}

Note: ID: first identification; RD: reidentification. 
linear positive ion mode with a laser frequency of $20 \mathrm{~Hz}$, LT: ISI $20 \mathrm{kV}$, IS2 $18.5 \mathrm{kV}$, lens $8.5 \mathrm{kV}$, PIE $250 \mathrm{~ns}$, no gating, range: 20-20,000 Da [6]. A small amount of one colony was spotted on the target plate, $1 \mu \mathrm{l}$ of $70 \%$ aqueous formic acid, and after drying, $1 \mu \mathrm{l}$ of MALDI matrix ( $\alpha$-cyano-4-hydroxycinannamic acid in $50 \%$ acetonitrile $/ 2.5 \%$ trifluoroacetic acid) were added to the spot. Interpretation of $\log$ score values was as follows: 0.000-1.699: unreliable identification; $1.700-$ 1.999: genus-level identification; $\geq 2.000$ : species-level identification. We applied B. fragilis ATCC 25285 and B. thetaiotaomicron ATCC 29742 strains as controls.

\section{Rapid ID $32 \mathrm{~A}$}

Twenty-one strains with contradictory results of identification and reidentification obtained by MALDI-TOF MS were checked by traditional biochemical test kit of rapid ID 32A method according to the manufacturer's instructions. Suspension with 4 McFarland turbidity was prepared from $48 \mathrm{~h}$ subculture in $2 \mathrm{ml}$ of sterile suspension medium and dropped $55 \mu \mathrm{l}$ into each cupule. The cupule for urease enzyme was overlaid with mineral oil. After covering the strips, they were incubated under aerobic conditions for $4 \mathrm{~h}$ at $37^{\circ} \mathrm{C}$. To nitrate and indole cupules, the appropriate reagents were added and these tests were read after $5 \mathrm{~min}$. Catalase production was also directly investigated with $15 \%$ hydrogen peroxide. The biochemical profile was analyzed by computer with a specific database (analytic profile index, version 3.2) provided by the manufacturer. B. fragilis ATCC 25285 and B. thetaiotaomicron ATCC 29742 were used as control strains.

\section{Reverse transcription polymerase chain reaction (RT-PCR)}

Twenty-one strains with contradictory results were identified by $16 \mathrm{~S}$ rRNA gene sequencing as well. DNA templates for PCR analyses were prepared as follows: one colony of each isolate was suspended in $100 \mu \mathrm{l}$ of distilled water and heated at $99.5{ }^{\circ} \mathrm{C}$ for $12 \mathrm{~min}$ in a dry bath. The RT-PCRs for amplification of $16 \mathrm{~S}$ rRNA gene were performed using $30 \mu \mathrm{l}$ total volumes, containing $15 \mu \mathrm{l}$ of $2 \times \mathrm{SYBR}$ Green qPCR Master Mix (BioTool, USA), $10.2 \mu \mathrm{l}$ water, $0.6 \mu \mathrm{l}$ of E8F (5'AGAGTTTGATCCTGGCTCAG-3') and E533R (5'-TIACCGIIICTICTGGCAC$3^{\prime}$ ) primers (concentrations: $\left.35-35 \mathrm{pmol} / \mu \mathrm{l}\right), 0.6 \mu \mathrm{l}$ ROX (BioTool Swiss AG, Switzerland), and $3 \mu \mathrm{l}$ of DNA templates. StepOne RT-PCR machine (Applied Biosystems, USA) was used for the PCR cycling and detection: $95^{\circ} \mathrm{C}$ for $10 \mathrm{~min}$, followed by 35 cycles of $95{ }^{\circ} \mathrm{C}$ for $15 \mathrm{~s}, 56^{\circ} \mathrm{C}$ for $20 \mathrm{~s}, 72^{\circ} \mathrm{C}$ for $30 \mathrm{~s}$, and 1 cycle of $72{ }^{\circ} \mathrm{C}$ for $75 \mathrm{~s}$ and a melting curve detection from 72 to $95{ }^{\circ} \mathrm{C}$. 
$16 S$ rRNA gene sequencing

The DNA amplicons from RT-PCRs (proportional scale up to $30 \mu \mathrm{l}$ ) were purified using the Gel/PCR DNA Fragment Extraction Kit (Geneaid Biotech Ltd., Taiwan). Purified templates were sequenced with ABI BigDye ${ }^{\circledR}$ Terminator version 3.1 kit in Series Genome Analyzer 3500 (Life Technologies, USA). The obtained sequencing data were analyzed by NCBI BLAST (http://www.ncbi.nim. nih.gov/blast/cgi) and leBiBi software (http://pbil.univ-lyon1.fr/bibi), the reliable identification level was set to $98.00 \%$.

\section{Results}

During the routine identification in local laboratories and reidentification in Szeged, out of 400 strains, $379(94.75 \%)$ were correctly identified to species level with the $\log$ score value of $\geq 2.000$ (log score value range: $2.020-2.525$, average $\log$ score value: 2.249 ) and the best $\log$ score values were chosen for analysis. Among the results of parallel MALDI-TOF MS reidentification measurements performed by three persons in Szeged, the best log score from the identification results was chosen, and the distribution of the identification results is summarized in Table I. MALDI-TOF MS reidentification results of 21 strains with $\log$ score value range of $1.855-2.458$ were confirmed by $16 \mathrm{~S}$ rRNA gene sequencing method and rapid ID 32A. These isolates with discrepancy according to the reidentification in Szeged are 4 B. fragilis and 17 non- $B$. fragilis strains (seven $B$. thetaiotaomicron, one $P$. distasonis, one $B$. cellulosilyticus, one $B$. salyersiae, one B. stercoris, one Bacteroides intestinalis, one B. vulgatus, two $B$. ovatus, and two $B$. nordii). The log score value of five strains among the examined 21 isolates with contradictory results was under 2.000 (1.993-1.802), and these strains belong to non- $B$. fragilis group. The same identification results with MALDI-TOF MS and sequencing were obtained in case of $15(71.42 \%)$ isolates. Excellent identification results $(>95.00 \%)$ were obtained with rapid ID $32 \mathrm{~A}$ only in case of eight strains $(8 / 21,31.01 \%)$ (Table II). The database of rapid ID 32A does not contain the biochemical profiles of B. cellulosilyticus, B. nordii, $B$. salyersiae, and Bacteroides xylanisolvens, for this reason, four strains were not acceptable, whereas $P$. distasonis strains SY2 were identified as Capnocytophaga sp. In comparison of identification results with MALDI-TOF MS and rapid ID 32A, we reported only five concordant results $(5 / 21,23.81 \%)$. The quality of sequencing results was $\geq 98.00 \%$, with the exception of strain SY9, which was identified with the level of $95.00 \%$. In case of Bacteroides strains SY9, SY64, and SY81, the MALDI-TOF MS and 16S rRNA gene sequencing 


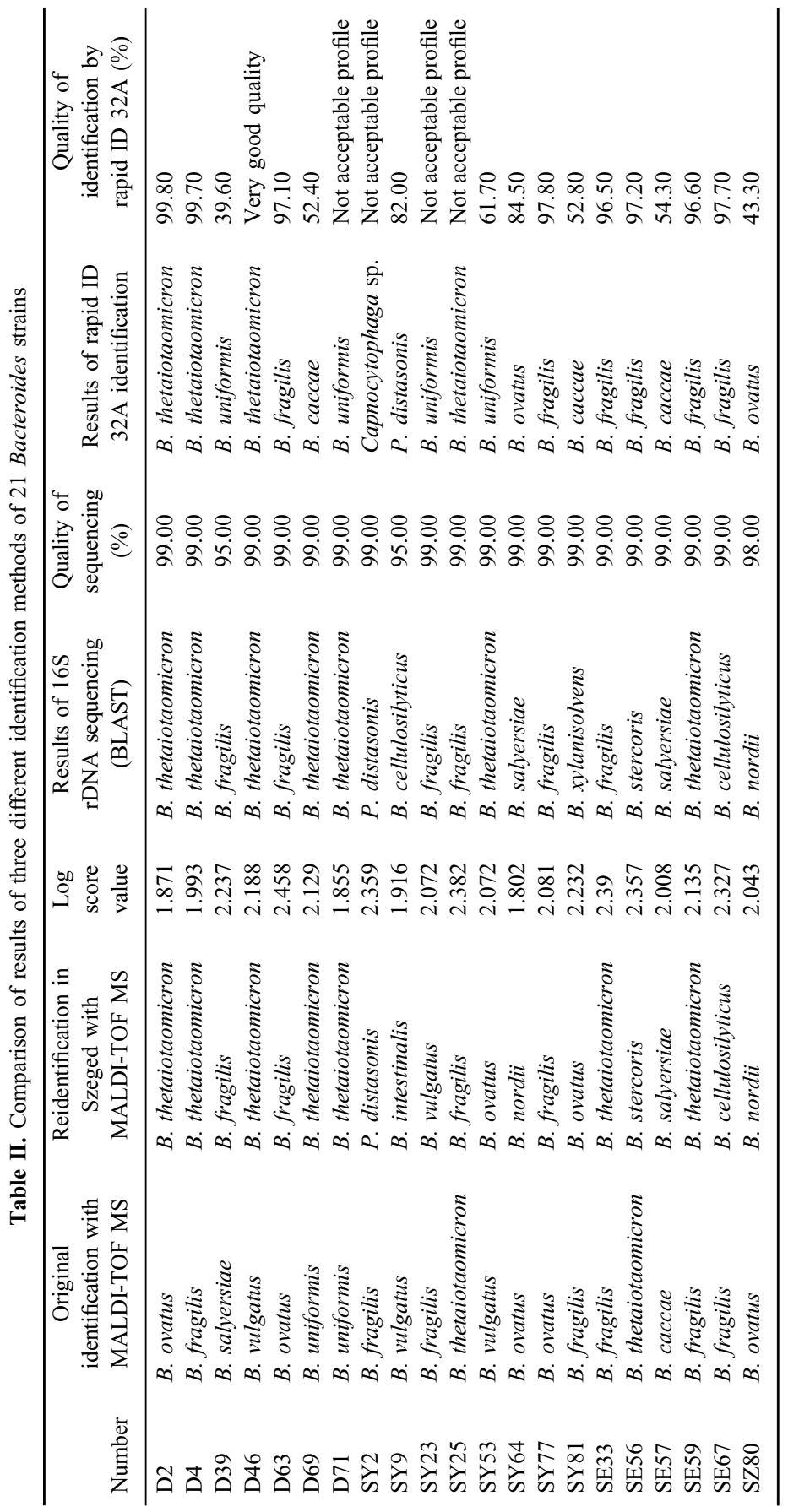


results were different (SY9: B. intestinalis/B. cellulosilyticus; SY64: B. nordii/ $B$. salyeriae; and SY81: B. ovatus/B. xylanisolvens), because these strains are phylogenetically closely related and the protein patterns of these species are so similar that makes identification by mass spectrometry difficult $[10,11]$. We accepted the sequencing results of $B$. fragilis SY23, B. thetaiotaomicron SY53, and $B$. fragilis SE33 strains. In the case of five isolates, the log score value of MALDI-TOF MS was bit lower than 2.00 (D2: 1.871; D4: 1.993; D71: 1.855; SY9:1.916; and SY64: 1.802).

\section{Discussion}

As several studies demonstrated the increasing rate of antibiotic-resistant strains among anaerobic bacteria, adequate species-level identification is getting very important. The traditional-automated methods have some limitations, e.g., the discrimination ability of biochemically similar strains is not sufficient. On the other hand, the results of identification may depend on the proper anaerobic environment and the deposited species in the library. Rapid ID 32A, as well as some other tests, cannot make difference between Gram-negative and Grampositive bacteria (one fits all). The database always needs to be improved and expanded with the newly recognized species. According to the literature data, the correct identification with preformed enzyme kits is only $78 \%-79 \%$ of the B. fragilis group isolates [6]. Another disadvantage of the biochemical test can be the length of incubation time [(rapid ID 32A: 4 h; API 20 A (bioMérieux): 24 h; remel rapid ID ANA II (Thermo Fisher Scientific, USA): $4 \mathrm{~h}$ ] and different incubation conditions, depending on the kind and the principle of kits. 16S rRNA gene sequencing is the most accurate method, but its complicated, timeconsuming, and expensive features inhibit the application in routine clinical microbiology. The MALDI-TOF MS system revolutionized and simplified the identification of various clinical isolates. This method is easy to perform within a short period of time and reproducible, and this has a high discriminatory power. This study demonstrated that $94.75 \%$ of Bacteroides isolates were correctly identified with Biotyper software 3.0. According to the $d n a J$, gyrB, hsp60, recA, rpoB, and 16S rRNA gene sequencing data, the phylogenetically related Bacteroides species classify to clades, e.g., species pairs: $B$. intestinalis/B. cellulosilyticus, B. nordii/B. salyersiae, and B. ovatus/B. xylanisolvens [10]. The differences among the results by MALDI-TOF MS and 16S rRNA gene sequencing can be explained with the classification in the same phylogenetical clade of Bacteroides strains SY9, SY64, and SY81. In the case of Bacteroides isolates SY23 (B. vulgatus/B. fragilis), SY53 (B. ovatus/B. thetaiotaomicron), and SE33 
(B. thetaiotaomicron/B. fragilis), we accepted the 16S rRNA sequencing results. Culebras et al. [6] reported that the accurate, species-level identification of Bacteroides strains with MALDI-TOF MS system is $87 \%$ in comparison with $16 \mathrm{~S}$ rRNA sequencing method. On the other hand, the rate of correct identification with rapid ID 32A method was 52.3\%. Nagy et al. [4] reported that the unequivocal identification rate of Bacteroides isolates was $98.60 \%$ of with MALDI-TOF MS. According to the data of study by Handal et al. [11], the species-level identification of Bacteroides and Gram-positive anaerobic cocci blood culture isolates is $86.6 \%$ by MALDI-TOF MS. This study proved the superiority of MALDI-TOF MS system to traditional-automated biochemical tests. For validation of the method, two types of measurements were applied: one person with three simultaneous measurements and three persons repeated the investigation with one measurement per person with the same conditions (strains and chemicals). Good reproducibility of MALDI-TOF MS identification method of Bacteroides species was proved with this study.

\section{Acknowledgements}

The authors would like to thank Katalin Kristof and Emese Juhasz for collecting Bacteroides strains for the investigations.

\section{Funding Sources}

None.

\section{Conflict of Interest}

None.

\section{Ethical Approval}

None.

\section{References}

1. Treviño, M., Areses, P., Peñalver, M. D., Cortizo, S., Pardo, F., Pérez del Molina, M. L., García-Riesta, C., Hernández, M., Llovo, J., Reguiero, B. J.: Susceptibility trends of Bacteroides fragilis group and characterization of carbapenemase-producing strains by automated REP-PCR and MALDI-TOF. Anaerobe 18, 37-43 (2012). 
2. Wexler, H. M.: Bacteroides: The good, the bad and the nitty-gritty. Clin Microbiol Rev 20 , 593-621 (2007).

3. Hsu, Y.-M. S., Burnham, C.-A. D.: MALDI-TOF MS identification of anaerobic bacteria: Assessment of pre-analytical variables and specimen preparation techniques. Diagn Microbiol Infect Dis 79, 144-148 (2014).

4. Nagy, E., Maier, T., Urban, E., Terhes, G., Kostrzewa, M.: Species identification of clinical isolates of Bacteroides by matrix-assisted laser-desorption/ionization time-of-flight mass spectrometry. Clin Microbiol Infect 15, 796-802 (2009).

5. Nagy, E., Urban, E., Nord, C. E.: Antimicrobial susceptibility of Bacteroides fragilis group isolates in Europe: 20 years of experience. Clin Microbiol Infect 17, 371-379 (2011).

6. Culebras, E., Rodríguez-Avial, I., Betriu, C., Gómez, M., Picazo, J. J.: Rapid identification of clinical isolates of Bacteroides species by matrix-assisted laser-desorption/ionisation time-of-flight mass spectrometry. Anaerobe 18, 163-165 (2012).

7. Jousimies-Somer, H., Summanen, P., Citron, D. M., Baron, E. J., Wexler, H. M., Finegold, S. M.: Wadsworth-KTL Anaerobic Bacteriology Manual, $6^{\text {th }}$ Edition. Star Publishing Company, Belmont, CA, 2002, p. 66.

8. Jousimies-Somer, H., Summanen, P.: Recent taxonomic changes and terminology update of clinically significant anaerobic Gram-negative bacteria (excluding spirochetes). Clin Infect Dis 35, 17-21 (2002).

9. Coltella, L., Mancinelli, L., Onori, M., Lucignano, B., Menichella, D., Sorge, R., Raponi, M., Mancini, R., Russo, C.: Advancement in the routine identification of anaerobic bacteria by MALDI-TOF mass spectrometry. Eur J Clin Microbiol 32, 1183-1192 (2013).

10. Sakamoto, M., Ohkuma, M.: Identification and classification of the genus Bacteroides by multilocus sequence analysis. Microbiology 157, 3388-3397 (2011).

11. Handal, N., Jørgensen, S. B., Tunsjø, H. S., Johnsen, B. O., Leegaard, T. M.: Anaerobic blood culture isolates in a Norwegian university hospital: Identification by MALDI-TOF MS vs 16S rRNA sequencing and antimicrobial susceptibility profiles. APMIS 123, 749-758 (2015). 